\title{
Laricitrin suppresses increased benzo(a)pyrene-induced lung tumor-associated monocyte-derived dendritic cell cancer progression
}

\author{
WEI-AN CHANG ${ }^{1,2^{*}}$, JEN-YU HUNG ${ }^{2,3^{*}}$, YING-MING TSAI $^{2,4}$, YA-LING HSU ${ }^{4}$, HUNG-HSING CHIANG ${ }^{1,5}$, \\ SHAH-HWA CHOU ${ }^{5}$, MING-SHYAN HUANG ${ }^{2,3}$ and PO-LIN KUO ${ }^{1,6,7}$ \\ ${ }^{1}$ Institute of Clinical Medicine, College of Medicine, Kaohsiung Medical University; ${ }^{2}$ Division of Pulmonary and Critical \\ Care Medicine, Kaohsiung Medical University Hospital; ${ }^{3}$ School of Medicine, College of Medicine, ${ }^{4}$ Graduate Institute of \\ Medicine, College of Medicine, Kaohsiung Medical University; ${ }^{5}$ Division of Chest Surgery, Department of Surgery, \\ Kaohsiung Medical University Hospital; ${ }^{6}$ Research Center for Environmental Medicine, \\ Kaohsiung Medical University, Kaohsiung 807; ${ }^{7}$ Institute of Medical Science and \\ Technology, National Sun Yat-Sen University, Kaohsiung 804, Taiwan, R.O.C.
}

Received February 14, 2015; Accepted January 13, 2016

DOI: $10.3892 / \mathrm{ol} .2016 .4153$

\begin{abstract}
Benzo(a)pyrene (BaP) stimulates lung cancer cells, promoting monocyte-derived dendritic cells to secrete soluble factors, including heparin binding-epidermal growth factor and C-X-C motif chemokine 5. The secretions from monocyte-derived dendritic cells stimulate the progression of lung cancer cells, including the migration and invasion of cells. To the best of our knowledge, these secretions remain unknown, and require additional study. The present study identified that treatment with BaP-H1395-tumor-associated dendritic cell-conditioned medium had the most marked effect on cell migration and invasion. This result may be associated with the female gender, stage 2 adenocarcinoma or mutation of the proto-oncogene B-Raf (BRAF), according to the cell line background. Laricitrin, a dietary flavonoid derivative present in grapes and red wine, suppresses certain factors and decreases the progression of lung cancer cells that are promoted by $\mathrm{BaP}$ in the lung cancer tumor microenvironment. The results of the present study suggest that prolonged
\end{abstract}

Correspondence to: Professor Ming-Shyan Huang, School of Medicine, College of Medicine, Kaohsiung Medical University, 100 Shiquan 1st Road, Kaohsiung 807, Taiwan, R.O.C.

E-mail: shyan310@gmail.com

Professor Po-Lin Kuo, Institute of Clinical Medicine, College of Medicine, Kaohsiung Medical University, 100 Shiquan 1st Road, Kaohsiung 807, Taiwan, R.O.C.

E-mail: kuopolin@seed.net.tw

${ }^{*}$ Contributed equally

Key words: benzo(a)pyrene, lung cancer, tumor microenvironment, laricitrin exposure to $\mathrm{BaP}$ exacerbates lung cancer, particularly in female lung cancer patients with the BRAF mutation, but that laricitrin may ameliorate this effect.

\section{Introduction}

Lung cancer is the leading cause of cancer mortality worldwide and is also associated with a poor prognosis (1). The tumor microenvironment has been demonstrated to be an important factor in cancer progression and drug resistance, as it may lead to dysregulated immune responses during tumor progression and the facilitation of tumor invasion (2). Tumor-associated dendritic cells (TADCs) are important in the tumor microenvironment, as they secrete numerous factors that promote lung cancer growth, migration, invasion and epithelial-to-mesenchymal transition (3). One factor, lung tumor-associated dendritic cell-derived resistin, has been indicated to promote cancer progression (4). Other lung TADC factors that have synergistic effects on cancer progression include heparin-binding epidermal growth factor-like growth factor and chemokine CXCL5 (5).

Benzo(a)pyrene $(\mathrm{BaP})$ is a carcinogenic polycyclic aromatic hydrocarbon that has been associated with lung cancer. BaP is found in cigarettes, food and automobile exhausts (6). BaP causes DNA adduct formation, which is the initiating event in carcinogenesis (7). BaP has also been demonstrated to promote A549 cell migration and invasion by upregulating Twist (8).

Flavonols are usually present in glycosidic forms and are synthesized in grape skin; therefore, they are also present in red wine (9). Flavonols are a subclass of flavonoid that have antioxidant properties and have a potential role in the prevention of cardiovascular disease (10). Flavonoids have also been demonstrated to have the potential to induce colorectal cancer cell apoptosis via the mitochondrial-mediated pathway (11). Flavonoids also suppress the growth of H460 and A549 cells by inducing cell cycle arrest in the $\mathrm{S}$ and $\mathrm{G} 2 / \mathrm{M}$ phases. Additionally, flavonoids also induce apoptosis in H460 and 
A549 cells (12). Laricitrin is a flavonol that is present predominantly as 3-glucoside (13). The present study investigated the association between laricitrin and the BaP-associated lung cancer tumor microenvironment.

\section{Materials and methods}

Chemicals. Laricitrin (Extrasynthese, Genay, France) was dissolved in dimethyl sulfoxide (DMSO; Sigma-Aldrich, St. Louis, MO, USA) and stored at $-20^{\circ} \mathrm{C}$. Control cultures contained the carrier solvent $0.1 \%$ DMSO.

Cell cultures and conditioned media (CM). The human lung adenocarcinoma H1395, H1975, H2087 and HCC2935 cell lines (catalog nos. ATCC CRL-5868, ATCC CRL-5908, ATCC CRL-5922 and ATCC CRL-2869, respectively) were purchased from the American Type Culture Collection (Manassas, VA, USA). The characteristics of the cell lines are reported in Table I. The cells were cultured in Gibco Roswell Park Memorial Institute (RPMI)-1640 medium (Thermo Fisher Scientific, Waltham, MA, USA) that contained $10 \%$ Gibco fetal bovine serum (Thermo Fisher Scientific). In order to obtain the various CM, the H1395, H1975, H2087 and HCC2935 cells $\left(2 \times 10^{6}\right.$ cells $/ 100 \mathrm{~mm}$ dish) were treated with or without BaP (Sigma-Aldrich) at a concentration of $10 \mu \mathrm{M}$ for $6 \mathrm{~h}$. Subsequent to washing and culturing for $24 \mathrm{~h}$, the CM of BaP-treated H1395, H1975, H2087 and HCC2935 cells (BaP-H1395-CM, BaP-H1975-CM, BaP-H2087-CM and BaP-HCC2935-CM, respectively) were harvested (Fig. 1A).

Isolation of $\mathrm{CD}^{+} 4^{+}$monocytes and differentiation of monocyte-derived dendritic cells (mdDCs). Monocytes were obtained from peripheral blood mononuclear cells (PBMCs) obtained from healthy, consenting donors. Mononuclear cells were isolated from the blood using the Ficoll-Hypaque gradient (GE Healthcare Life Sciences, Little Chalfont, UK). CD14 ${ }^{+}$ monocytes were purified using MACS MicroBeads CD14 ${ }^{+}$ monoclonal antibody-conjugated magnetic beads (Miltenyi Biotec GmbH, Bergisch Gladbach, Germany), according to the manufacturer's protocol. mdDCs were generated by culturing $\mathrm{CD}_{1}{ }^{+}$monocytes in RPMI-1640 containing FBS and $20 \mathrm{ng} / \mathrm{ml}$ granulocyte macrophage-colony-stimulating factor (GM-CSF) and $10 \mathrm{ng} / \mathrm{ml}$ interleukin-4 (IL-4) (R\&D Systems, Inc., Minneapolis, MN, USA) for 5 days. The medium was replaced with fresh medium containing GM-CSF and IL-4 on day 3. For the maturation of DCs, immature mdDCs were stimulated with lipopolysaccharide (LPS; $100 \mathrm{ng} / \mathrm{ml}$; Sigma-Aldrich) subsequent to priming with interferon- $\gamma$ (IFN- $\gamma$; EMD Millipore, Billerica, MA, USA) for $3 \mathrm{~h}$.

H1395-TADCs, Bap-H1395-TADCs, H1975-TADCs, BaP-H1975-TADCs, H2087-TADCs, BaP-H2087-TADCs, HCC2935-TADCs or BaP-HCC2935-TADCs were generated by culturing $\mathrm{CD}_{1} 4^{+}$monocytes in RPMI-1640 medium containing FBS,IL-4 and GM-CSF, with 20\% H1395-CM,BaP-H1395-CM, H1975-CM, BaP-H1975-CM, H2087-CM, BaP-H2087-CM, HCC2935-CM or BaP-HCC2935-CM. The cell culture was then stimulated with LPS, subsequent to priming with IFN- $\gamma$ for $3 \mathrm{~h}$. Subsequent to washing, the supernatants were collected and identified as H1395-TADC-CM BaP-H1395-TADC-CM, H 1975 -T A D C-CM , B a P-H 1975 - T A D C - CM, 

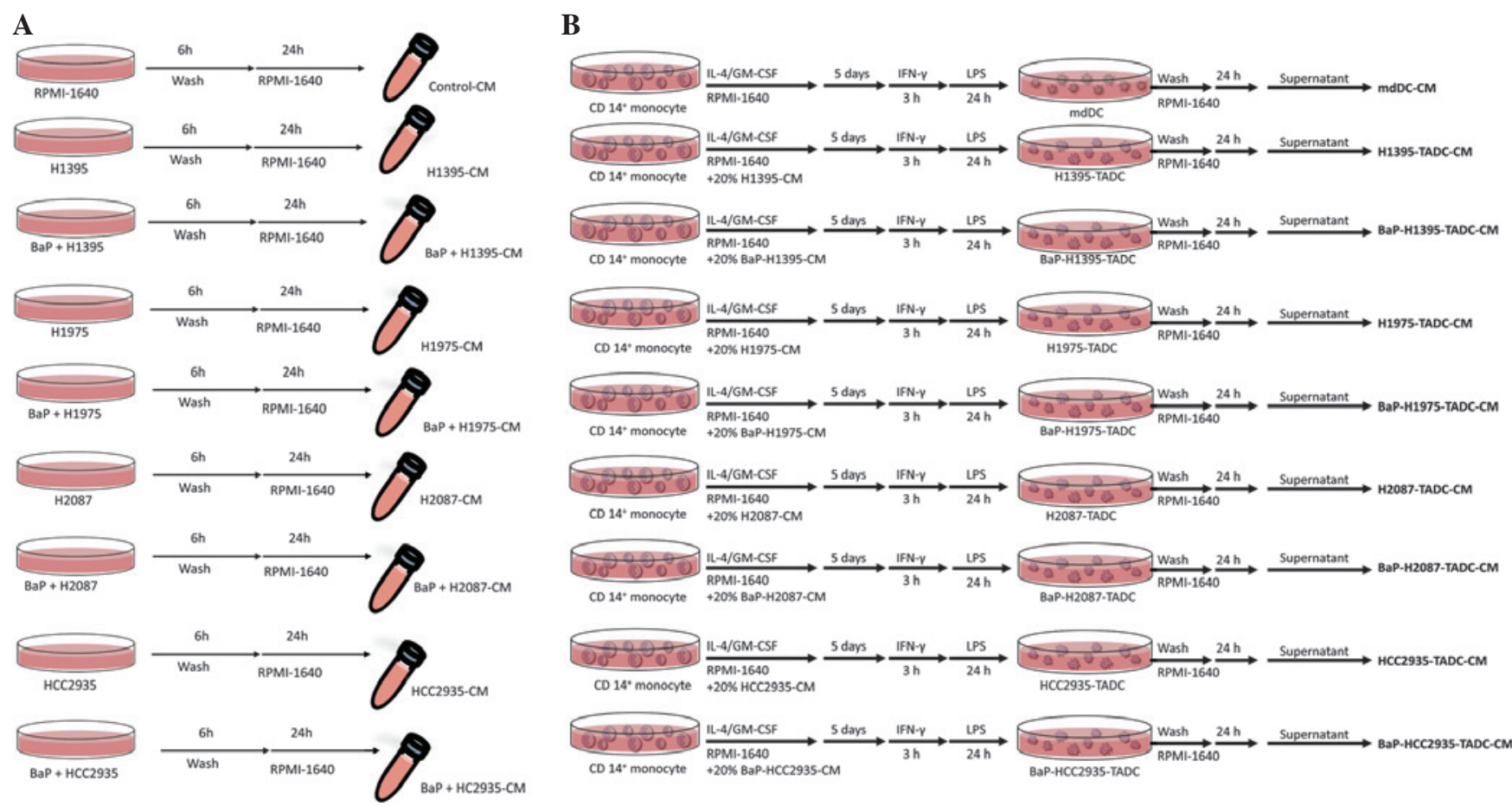

Figure 1. Flow chart of the production of various CM. (A) Flow chart of the production of control-CM, H1395-CM, BaP-H1395-CM, H1975-CM, BaP-H1975-CM, H2087-CM, BaP-H2087-CM, HCC2935-CM, and BaP-HCC2935-CM. (B) Flow chart of the production of mdDC-CM, H1395-TADC-CM, BaP-H1395-TADC-CM, H1975-TADC-CM, BaP-H1975-TADC-CM, H2087-TADC-CM, BaP-H2087-TADC-CM, HCC2935-TADC-CM and BaP-HCC2935-TADC-CM. CM, conditioned media; BaP, benzo(a)pyrene; mcDC, monocyte-derived dendritic cell; TADC, tumor-associated dendritic cell; IL-4, interleukin 4; GM-CSF, granulocyte macrophage-colony-stimulating factor; IFN- $\gamma$, interferon- $\gamma$; LPS, lipopolysaccharide.

H 2087 -T A D C-CM, B a P-H $2087-\mathrm{TA} \mathrm{DC}-\mathrm{CM}$, HCC2935-TADC-CM or BaP-HCC2935-TADC-CM (Fig. 1B). The Institutional Review Board (IRB) of Kaohsiung Medical University Hospital (Kaohsiung, Taiwan) approved the protocol of the present study (IRB nos., KMUH-IRB-990345, KMUH-IRB-20110377 and KMUH-IRB-20130054), and all participants provided written informed consent in accordance with the Declaration of Helsinki (5).

Cell proliferation. The cells were plated in 96-well culture plates. Following $24 \mathrm{~h}$ incubation, the cells were treated with vehicle mdDC-CM or various $\mathrm{CM}$ for $72 \mathrm{~h}$. The effects of H1395-TADC-CM and BaP-H1395-TADC-CM on the proliferation of H1395 cells, H1975-TADC-CM and BaP-H1975-TADC-CM on the proliferation of H1975 cells, H2087-TADC-CM and BaP-H2087-TADC-CM on the proliferation of $\mathrm{H} 2087$ cells, and HCC2935-TADC-CM and $\mathrm{BaP}-\mathrm{HCC} 2935-\mathrm{TADC}-\mathrm{CM}$ on the proliferation of HCC2935 cells were assessed using a water-soluble tetrazolium salt-1 (WST-1) assay (Clontech Laboratories, Inc., Mountain View, CA, USA) subsequent to $72 \mathrm{~h}$ incubation. Cell proliferation was determined by Premixed WST-1 Cell Proliferation reagent (Clontech Laboratories, Inc.) in accordance with the manufacturer's protocol. Then, the H1395, H1975, H2087 or HCC2935 cells were treated with the vehicle control or $10 \mu \mathrm{M}$ of BaP for $6 \mathrm{~h}$.

Cell migration and invasion assays. The cell migration and invasion assays were conducted using QCM $^{\mathrm{TM}}$ 24-well Cell Migration and Invasion Assay kits (EMD Millipore). The effects of H1395-TADC-CM and BaP-H1395-TADC-CM on the migration of H1395 cells, H1975-TADC-CM and BaP-H1975-TADC-CM on the migration of H1975 cells, H2087-TADC-CM and BaP-H2087-TADC-CM on the migration of $\mathrm{H} 2087$ cells, and HCC2935-TADC-CM and BaP-HCC2935-TADC-CM on the migration of HCC2935 cells were quantified using the QCM 24-well cell migration assay. Briefly, the cells were seeded onto the migration chamber and mdDC-CM or a $20 \%$ concentration of the various media was added to the bottom wells to act as a chemoattractant for $24 \mathrm{~h}$.

The QCM 24-well cell invasion assay was used to quantify the effect of H1395-TADC-CM and BaP-H1395-TADC-CM on the invasion of H1395 cells, H1975-TADC-CM and BaP-H1975-TADC-CM on the invasion of H1975 cells, H2087-TADC-CM and BaP-H2087-TADC-CM on the invasion of H2087 cells, and HCC2935-TADC-CM and BaP-HCC2935-TADC-CM on the invasion of HCC2935 cells. A $20 \%$ concentration of the aforementioned media acted as a chemoattractant for $48 \mathrm{~h}$.

At the end of treatment in the two assays, the cells were stained with CyQuant GR dye (part of the QCM 24-well Cell Migration and Invasion Assay kit; EMD Millipore) in cell lysis buffer for $15 \mathrm{~min}$ at room temperature. The fluorescence of the migrated and invaded cells was read using a fluorescence plate reader (FLx800; Bio-Tek Instruments, Inc., Winooski, VT, USA) at excitation/emission wavelengths of $485 / 520 \mathrm{~nm}$.

Laricitrin treatments. The protocol for assessing the effect of laricitrin involved the aforementioned isolation, proliferation, migrationandinvasionsteps, withaslightmodification.Briefly, the mdDCs,H1395-TADCs,Bap-H1395-TADCs,HCC2935-TADCs 

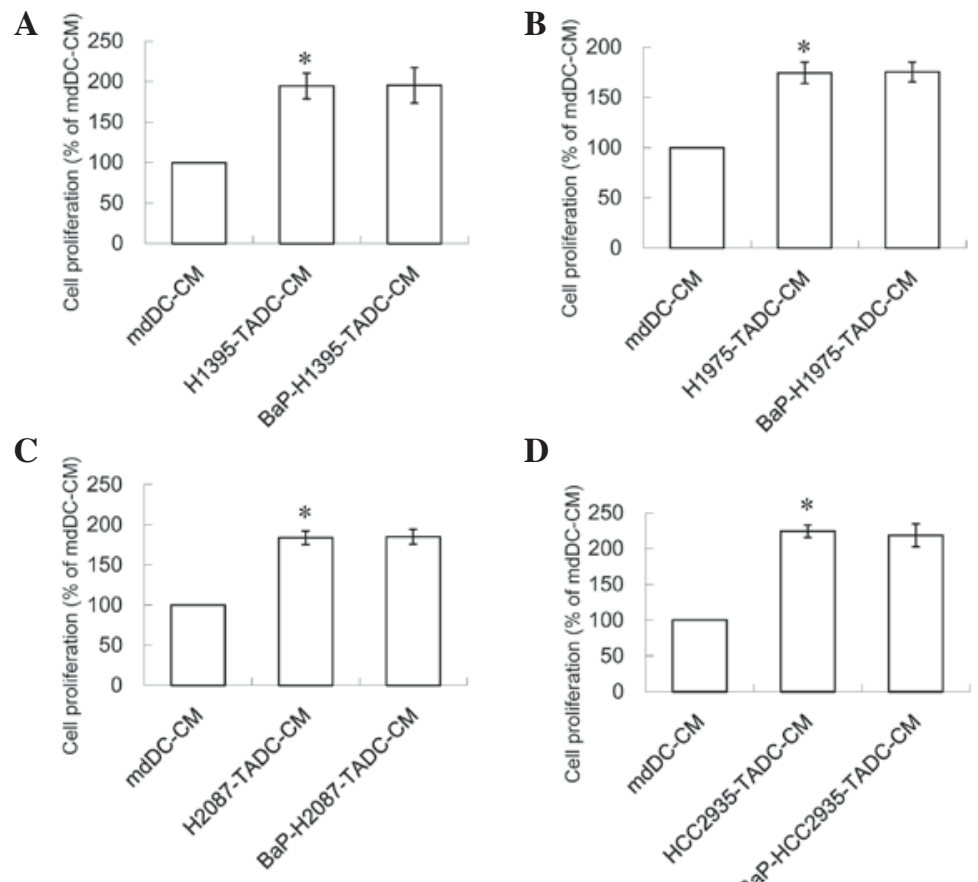

D

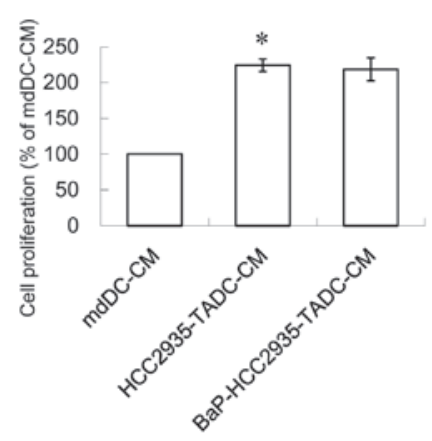

Figure 2. CM of BaP-treated lung cancer cells did not cause mdDCs to enhance lung cancer cell proliferation. (A) Comparison between the proliferation of H1395 cells cultured in BaP-H1395-TADC-CM and H1395-TADC-CM. (B) Comparison between the proliferation of H1975 cells cultured in BaP-H1975-TADC-CM and H1975-TADC-CM. (C) Comparison between the proliferation of H2087 cells cultured in BaP-H2087-TADC-CM and H2087-TADC-CM. (D) Comparison between the proliferation of HCC2935 cells cultured in BaP-HCC2935-TADC-CM and HCC2935-TADC-CM. The data are expressed as the mean \pm standard deviation of 3 independent experiments. ${ }^{*} \mathrm{P}<0.05$ vs. mdDC-CM treatment. CM, conditioned media; BaP, benzo(a)pyrene; mdDC, monocyte-derived dendritic cells; TADC, tumor-associated dendritic cell.

or BaP-HCC2935-TADCs were derived by culturing CD14 ${ }^{+}$ monocytes in RPMI-1640 medium containing FBS, IL-4 and GM-CSF, with or without a 20\% concentration of H1395-CM, BaP-H1395-CM, HCC2935-CM or BaP-HCC2935-CM, for $72 \mathrm{~h}$. For the maturation of DCs, immature mdDCs were stimulated with $100 \mathrm{ng} / \mathrm{ml}$ LPS subsequent to priming with IFN- $\gamma$ for 3 h. mdDCs, H1395-TADCs, Bap-H1395-TADCs, HCC2935-TADCs or BaP-HCC2935-TADCs were then pretreated with or without $2 \mu \mathrm{M}$ laricitrin for $1 \mathrm{~h}$. Subsequently, mdDC-CM, H1395-TADC-CM, BaP-H1395-TADC-CM, HCC2935-TADC-CM or BaP-HCC2935-TADC-CM, with or without laricitrin, were added to $\mathrm{H} 1395$ or HCC2935 cells, as appropriate, for another $72 \mathrm{~h}$. Cell proliferation was assessed using a WST-1 assay. H1395 and HCC2935 cells were seeded into the top Transwell insert, and the various CMs of mdDCs, laricitrin-treated mdDCs, H1395-TADCs, laricitrin-treated H1395-TADCs, BaP-H1395-TADCs, laricitrin-treated Bap-H1395-TADCs, HCC2935-TADCs, laricitrin-treated HCC2935-TADCs, BaP-H2935-TADCs or laricitrin-treated Bap-HCC2935-TADCs were added to the bottom chamber as chemoattractants for $24 \mathrm{~h}$ (for migration) or $48 \mathrm{~h}$ (for invasion). The migratory and invasive abilities of the H1395 and HCC2935 cells were quantified by QCM 24-well Cell Migration and Invasion assay.

Statistical analysis. The data were expressed as the mean \pm standard error. Statistical comparisons were made using one-way analysis of variance with post-hoc Tukey's test. Significant differences between the means of the two test groups were analyzed by Student's $t$-test. $\mathrm{P}<0.05$ was considered to indicate a statistically significant difference.

\section{Results}

Subsequent to exposure to BaP, lung cancer cells affect $m d D C s$, which then contributes to lung cancer progression by increasing cancer cell migration and invasion. To investigate the effect of $\mathrm{BaP}$ on tumor progression in the lung cancer tumor microenvironment, the effects of BaP-H1395-TADC-CM, BaP-H1975-TADC-CM, BaP-H2087-TADC-CM and $\mathrm{BaP}-\mathrm{HCC} 2935-\mathrm{CM}$ on the proliferation, migration and invasion of lung cancer cells were examined. A concentration of $20 \%$ of the aforementioned media increased the proliferation of lung cancer cells. This effect is similar to that in H1395, H1975, H2087 or HCC2935 cells treated with $10 \mu \mathrm{M} \mathrm{BaP}$. Notably, the proliferation effect was not increased when these cells were treated with BaP (Fig. 2). Compared with H1395-TADC-CM, BaP-H1395-TADC-CM did not enhance H1395 cell proliferation (Fig. 2A). Compared with H1975-TADC-CM, BaP-H1975-TADC-CM did not enhance H1975 cell proliferation (Fig. 2B). Compared with H2087-TADC-CM, BaP-H2087-TADC-CM did not enhance H2087 cell proliferation (Fig. 2C). Compared with HCC2935-TADC-CM, BaP-HCC2935-TADC-CM did not enhance HCC2935 cell proliferation (Fig. 2D). However, a $20 \%$ concentration of H1395-TADC-CM, H1975-TADC-CM, H2087-TADC-CM or HCC2935-TADC-CM was indicated to induce lung cancer cell migration and invasion, and a $20 \%$ concentration of the aforementioned media may additionally increase this effect (Figs. 3 and 4). It was found that BaP-H1395-TADC-CM enhanced H1395 cell migration and invasion compared with H1395-TADC-CM (Figs. 3A and 4A). BaP-H1975-TADC-CM enhanced H1975 
A

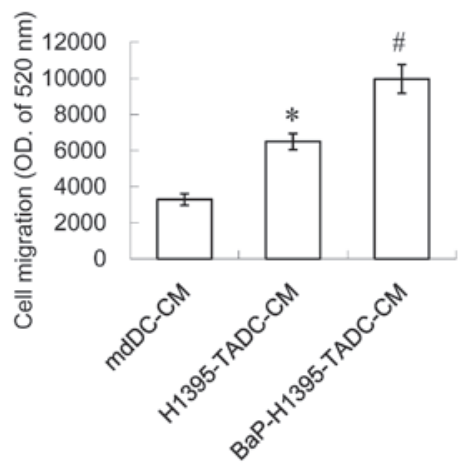

C

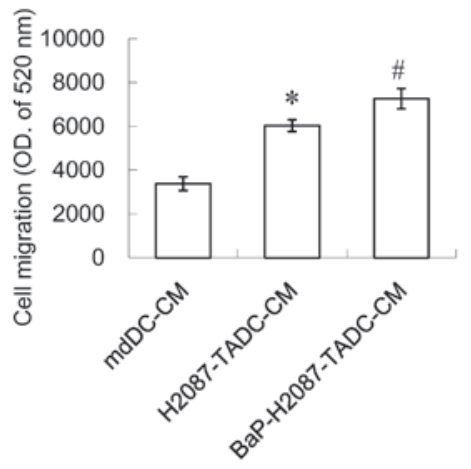

B

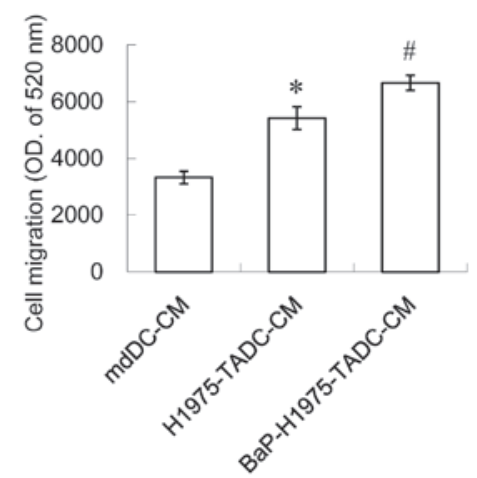

D

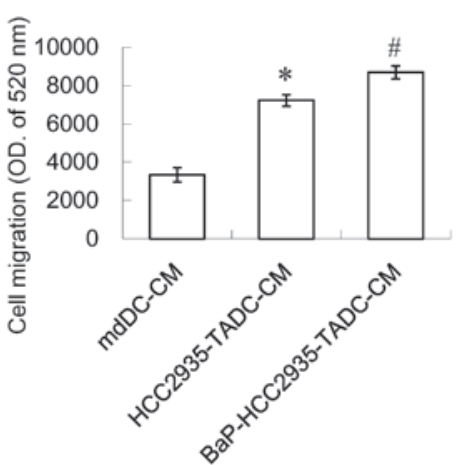

Figure 3. CM of BaP-treated lung cancer caused mdDCs to enhance lung cancer cell migration. (A) Comparison between the migration of H1395 cells cultured in BaP-H1395-TADC-CM and H1395-TADC-CM. (B) Comparison between the migration of H1975 cells cultured in BaP-H1975-TADC-CM and H1975-TADC-CM. (C) Comparison between the migration of H2087 cells cultured in BaP-H2087-TADC-CM and H2087-TADC-CM. (D) Comparison between the migration of HCC2935 cells cultured in BaP-HCC2935-TADC-CM and HCC2935-TADC-CM. The data are expressed as the mean \pm standard deviation of 3 independent experiments. "P<0.05 vs. mdDC-CM treatment; " $\mathrm{P}<0.05$ vs. TADC-CM treatment. CM, conditioned media; BaP, benzo(a)pyrene; mdDC, monocyte-derived dendritic cell; TADC, tumor-associated dendritic cell; OD, optical density.

A

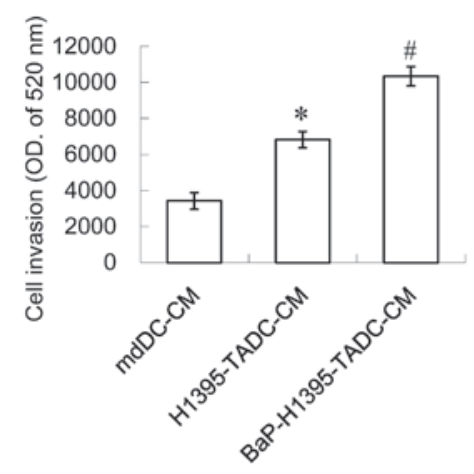

C

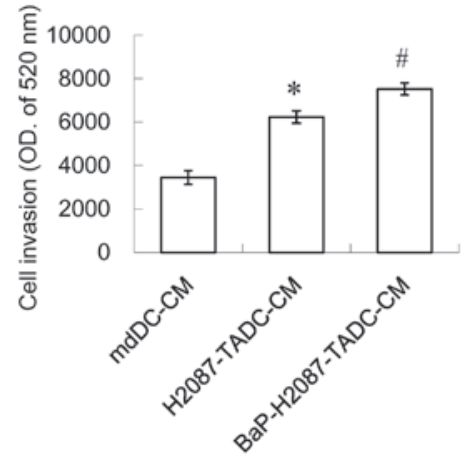

B

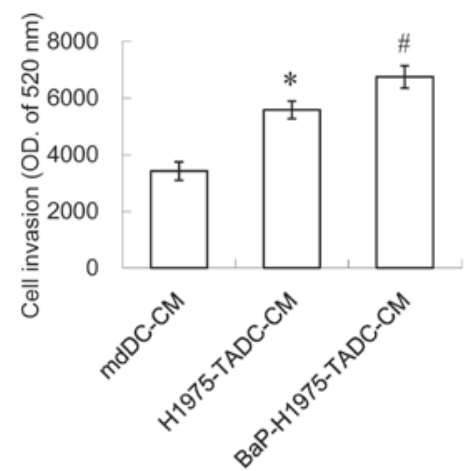

D

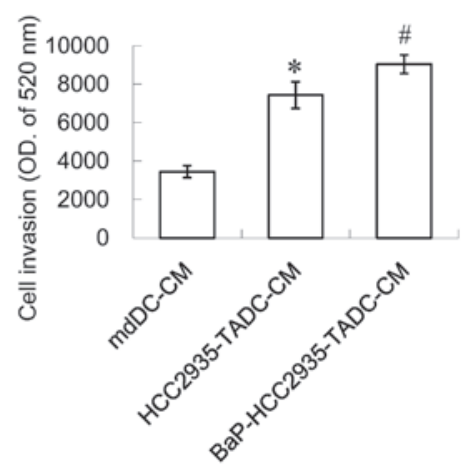

Figure 4. CM of BaP-treated lung cancer caused mdDCs to exacerbate lung cancer cell invasion. (A) Comparison between the invasion of H1395 cells cultured in BaP-H1395-TADC-CM and H1395-TADC-CM. (B) Comparison between the invasion of H1975 cells cultured in BaP-H1975-TADC-CM and H1975-TADC-CM. (C) Comparison between the invasion of H2087 cells cultured in BaP-H2087-TADC-CM and H2087-TADC-CM. (D) Comparison between the invasion of HCC2935 cells cultured in BaP-HCC2935-TADC-CM and HCC2935-TADC-CM. The data are expressed as the mean \pm standard deviation of 3 independent experiments. " $\mathrm{P}<0.05$ vs. mdDC-CM treatment; " $\mathrm{P}<0.05$ vs. TADC-CM treatment. CM, conditioned media; BaP, benzo(a)pyrene; mdDC, monocyte-derived dendritic cell; TADC, tumor-associated dendritic cell; OD, optical density. 

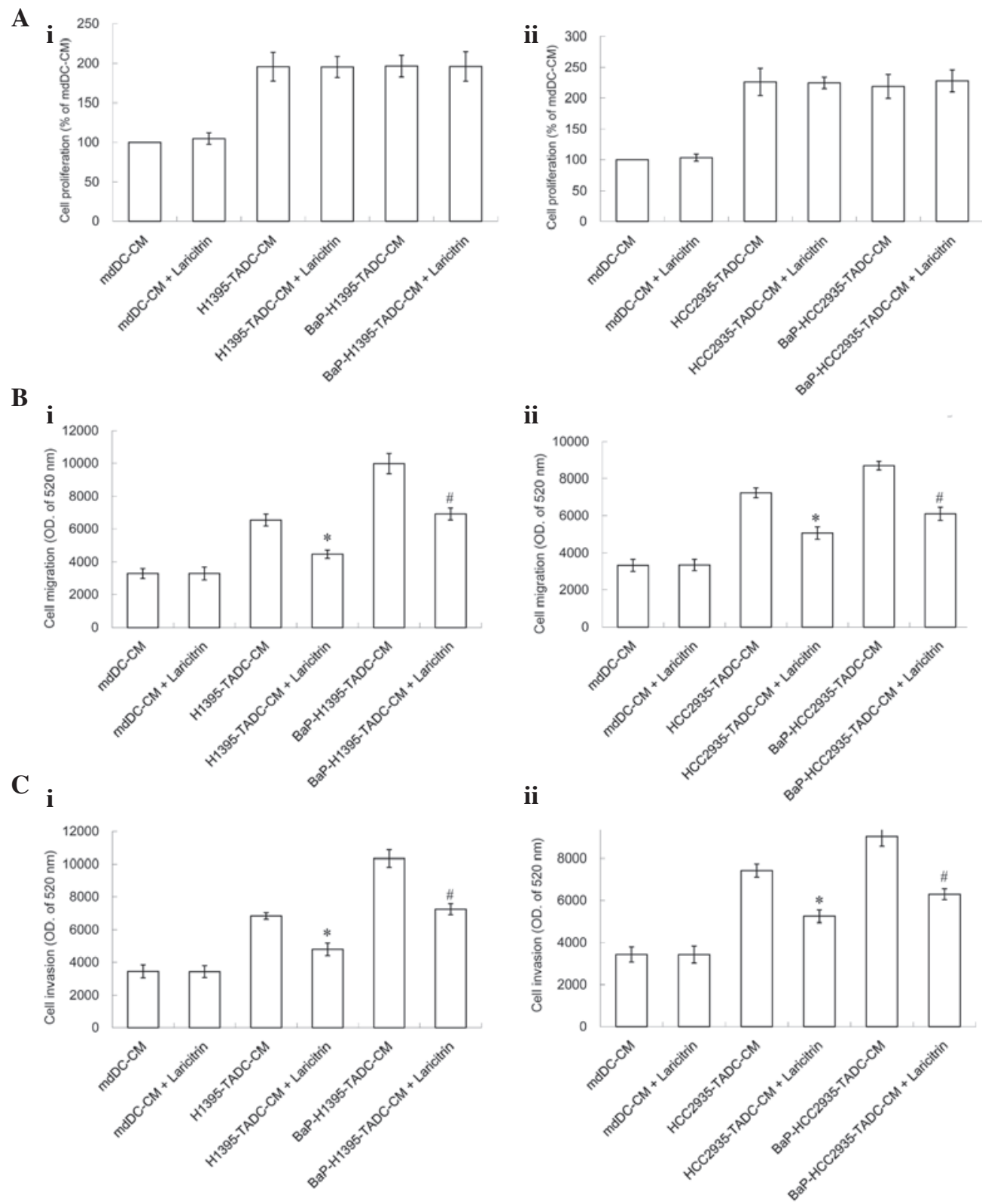

Figure 5. Laricitrin suppresses BaP-mediated cancer aggravation in the lung cancer tumor microenvironment. The effect of laricitrin on the (A) proliferation, (B) migration and (C) invasion of i) $\mathrm{H} 1395$ and ii) HCC2935 cells in the TADC-CM or BaP-TADC-CM cultures. The data are expressed as the mean \pm standard deviation of 3 independent experiments. ${ }^{\mathrm{P}}<0.05$ vs. TADC-CM treatment; ${ }^{*} \mathrm{P}<0.05$ vs. BaP-TADC-CM treatment. CM, conditioned medium; BaP, benzo(a)pyrene; mdDC, monocyte-derived dendritic cell; TADC, tumor associated dendritic cell; OD, optical density.

cell migration and invasion compared with H1975-TADC-CM (Figs. 3B and 4B). BaP-H2087-TADC-CM enhanced H2087 cell migration and invasion compared with H2087-TADC-CM (Figs. 3C and 4C). In addition, BaP-HCC2935-TADC-CM enhanced HCC2935 cell migration and invasion compared with HCC2935-TADC-CM (Figs. 3D and 4D). Therefore, the proliferation effect may not be increased; however, the migration and invasion effects increase in cancer cells with BaP-treatment. Comparing each TADC-CM with mdDC-CM, HCC2935-TADC-CM revealed that had the most marked effect on cancer proliferation, migration and invasion. Comparison of the BaP-treated TADC-CMs with each TADC-CM revealed that BaP-H1395-TADC-CM demonstrated the most marked effect on promoting cancer cell migration and invasion.
Laricitrin suppresses BaP-induced lung tumor-associated monocyte-derived dendritic cell-increased cancer progression in the lung cancer tumor microenvironment. Compared with cell lines not treated with BaP, HCC2935-TADC-CM demonstrated the most marked effect on the proliferation, migration and invasion of lung cancer cells. However, BaP-H1395-TADC-CM exerted the strongest effect on the migration and invasion of lung cancer cells (Figs. 3 and 4). Therefore, the HCC2935 and H1395 cell lines were selected as models for developing antidotes against BaP-associated cancer aggravation in the lung cancer tumor microenvironment.

The effect of laricitrin, a dietary flavonoid derivative present in grapes and red wine, on BaP-induced cancer progression was then assessed. The results in Fig. 5 revealed that, although laricitrin did not inhibit the proliferation of 


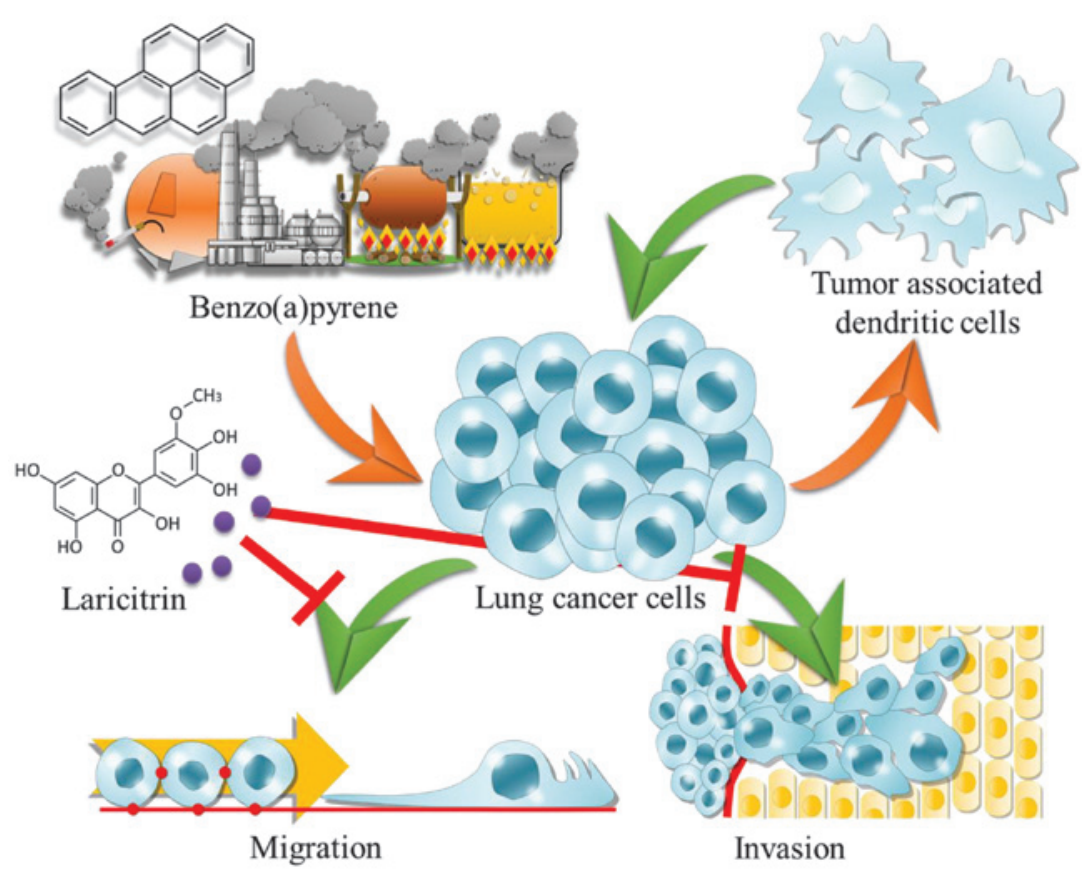

Figure 6. Laricitrin reverses benzo(a)pyrene-associated lung cancer aggravation in the lung cancer tumor microenvironment.

lung cancer cells in the lung cancer tumor microenvironment (Fig. 5A), it did inhibit the lung cancer cell migration induced by H1395-TADC-CM and HCC2935-TADC-CM (Fig. 5B). In addition, laricitrin suppressed the lung cancer cell invasion that was induced by BaP-H1395-TADC-CM and BaP-HCC2935-TADC-CM (Fig. 5C).

\section{Discussion}

Lung cancer is currently the leading cause of cancer-associated mortality worldwide. BaP has been demonstrated to induce lung cancer development; however, the role of $\mathrm{BaP}$ in the lung cancer tumor microenvironment remains unclear. Exposure to $\mathrm{BaP}$ may not be completely avoided; however, numerous factors may affect the deleterious effects of $\mathrm{BaP}$, such as gender, epidermal growth factor receptor (EGFR) mutation and proto-oncogene B-Raf (BRAF) mutation. The present study investigated the association between certain factors and the effect of $\mathrm{BaP}$ on lung cancer progression. Table I shows the background of the cell lines used in the present study (14-28). A previous study using CD-1 mice indicated that female mice are more susceptible to the carcinogenic effects of $\mathrm{BaP}$ compared with males (29). Therefore, being female may increase the risk of BaP-induced lung cancer. BRAF mutation is another risk factor for non-small cell lung cancer and adenocarcinoma of the lungs (30). A previous study performed using BRAF V600E mutant mice revealed that the expression of the BRAF mutation leads to adenocarcinoma development and is essential for tumor maintenance (31). EGFR mutation is also a risk factor for adenocarcinoma, as EGFR maintains the malignant phenotype of lung cancer cells (32). Comparison between invasive adenocarcinoma and preinvasive adenocarcinoma tissue samples indicated that there is an increased frequency of EGFR mutation in invasive adenocarcinoma, and an increased frequency of the BRAF mutation in preinvasive adenocarcinoma (33). Although
EGFR mutation is not an independent prognostic factor (34), BRAF mutation is associated with poor disease-free survival and poor overall survival rates (35). Female gender, late-stage lung cancer and EGFR and BRAF mutations are risk factors for BaP-induced lung cancer progression. In addition, BRAF mutation is a greater risk factor compared with EGFR mutation.

Comparison between the BaP-treated TADC-CMs and each TADC-CM indicated that BaP-H1395-TADC-CM had the most marked effect on promoting cancer cell migration and invasion, with an increase of 1.5 fold, compared with the other cell lines, which increased $\sim 1.2$ fold. This result may be due to $\mathrm{H} 1395$ cells possessing the greatest number of risk factors, as they were obtained from a female smoker with BRAF mutation (14-18). Comparison between each TADC-CM and mdDC-CM indicated that HCC2935-TADC-CM had the most marked effect on the proliferation, migration and invasion of cancer cells. The HCC2935 cell line was established from the pleural effusion cells of an adenocarcinoma patient (18-22). Lung cancer with malignant pleural effusion indicates terminal-stage disease (36). Therefore, HCC2935 cells may possess increased potential for metastasis compared with other cell lines.

To the best of our knowledge, the present study is the first to investigate the association between $\mathrm{BaP}$ and lung tumor-associated monocyte-derived dendritic cell-increased cancer aggravation in various cell lines. The present study has two novel findings: i) That lung tumor-associated monocyte-derived dendritic cells, following exposure to $\mathrm{BaP}$, contribute to cancer progression by increasing cancer cell migration and invasion; and ii) that laricitrin, a dietary flavonoid derivative present in grapes and red wine, reverses BaP-mediated lung cancer aggravation (Fig. 6). BaP exacerbates cancer cell migration and invasion in the lung cancer tumor microenvironment. Laricitrin may reverse this effect. Notably, the H1395 cell line demonstrated the most marked enhancement of migration and invasion following exposure to 
BaP-H1395-TADC-CM, and possessed the greatest number of risk factors. This finding suggests that $\mathrm{BaP}$ may be able to induce cancer cell migration and invasion without promoting proliferation in patients with the highest number of risk factors. Therefore, patients with lung cancer that possess numerous risk factors and are in contact with BaP may demonstrate an increased possibility of metastasis and invasion. In conclusion, the development of a drug with the potential for treating $\mathrm{BaP}-$ induced cancer progression is vitally important.

\section{Acknowledgements}

The present study was supported by the National Science Council (grant nos., NSC 101-2628-B-037-001-MY3 and NSC 101-2320-B-037-043-MY3), Ministry of Science and Technology (grant nos., MOST 104-2320-B-037-014-MY3, MOST 103-2314-B-037-052 and MOST 103-2320-B-037-032), Kaohsiung Medical University 'Aim for the Top 500 Universities Grant' (grant no., KMU-DT103008), Kaohsiung Medical University 'Aim for the Top Universities Grant' (grant nos., KMU-TP103A19 and KMU-TP103A20) and the Kaohsiung Medical University Hospital Research Foundation (grant no., KMUH103-3M04). The authors thank the Center for Research Resources and Development of Kaohsiung Medical University for support with the instrumentation.

\section{References}

1. Jemal A, Siegel R, Ward E, Hao Y, Xu J and Thun MJ: Cancer statistics, 2009. CA Cancer J Clin 59: 225-249, 2009.

2. Tsai MJ, Chang WA, Huang MS and Kuo PL: Tumor microenvironment: A new treatment target for cancer. ISRN Biochem 2014: 351959, 2014.

3. Hsu YL, Huang MS, Cheng DE, Hung JY, Yang CJ, Chou SH and Kuo PL: Lung tumor-associated dendritic cell-derived amphiregulin increased cancer progression. J Immunol 187: 1733-1744, 2011.

4. Kuo CH, Chen KF, Chou SH, Huang YF, Wu CY, Cheng DE, Chen YW, Yang CJ, Hung JY and Huang MS: Lung tumor-associated dendritic cell-derived resistin promoted cancer progression by increasing Wolf-Hirschhorn syndrome candidate 1/Twist pathway. Carcinogenesis 34: 2600-2609, 2013.

5. Kuo PL, Huang MS, Hung JY, Chou SH, Chiang SY, Huang YF, Yang CJ, Tsai MJ, Chang WA and Hsu YL: Synergistic effect of lung tumor-associated dendritic cell-derived HB-EGF and CXCL5 on cancer progression. Int J Cancer 135: 96-108, 2014.

6. Alexandrov K, Rojas M and Satarug S: The critical DNA damage by benzo(a)pyrene in lung tissues of smokers and approaches to preventing its formation. Toxicol Lett 198: 63-68, 2010.

7. Zuo J, Brewer DS, Arlt VM, Cooper CS and Phillips DH: Benzo pyrene-induced DNA adducts and gene expression profiles in target and non-target organs for carcinogenesis in mice. BMC Genomics 15: 880, 2014.

8. Wang Y, Zhai W, Wang H, Xia X and Zhang C: Benzo(a)pyrene promotes A549 cell migration and invasion through up-regulating Twist. Arch Toxicol 89: 451-458, 2015.

9. Mattivi F, Guzzon R, Vrhovsek U, Stefanini M and Velasco R Metabolite profiling of grape: Flavonols and anthocyanins. J Agric Food Chem 54: 7692-7702, 2006.

10. Kozłowska A and Szostak-Wegierek D: Flavonoids - food sources and health benefits. Rocz Panstw Zakl Hig 65: 79-85, 2014.

11. Wang CZ, Calway TD, Wen XD, Smith J, Yu C, Wang Y, Mehendale SR and Yuan CS: Hydrophobic flavonoids from Scutellaria baicalensis induce colorectal cancer cell apoptosis through a mitochondrial-mediated pathway. Int J Oncol 42: 1018-1026, 2013

12. Tsui KC, Chiang TH, Wang JS, Lin LJ, Chao WC, Chen BH and Lu JF: Flavonoids from Gynostemma pentaphyllum exhibit differential induction of cell cycle arrest in H460 and A549 cancer cells. Molecules 19: 17663-17681, 2014.
13. Castillo-Muñoz N, Gómez-Alonso S, García-Romero E and Hermosín-Gutiérrez I: Flavonol profiles of Vitis vinifera red grapes and their single-cultivar wines. J Agric Food Chem 55: 992-1002, 2007.

14. NCI-Navy Medical Oncology Branch cell line supplement. J Cell Biochem (Suppl) 24: 1-291, 1996

15. Naoki K, Chen TH, Richards WG, Sugarbaker DJ and Meyerson M: Missense mutations of the BRAF gene in human lung adenocarcinoma. Cancer Res 62: 7001-7003, 2002.

16. Koivunen JP, Kim J, Lee J, et al: Mutations in the LKB1 tumour suppressor are frequently detected in tumours from Caucasian but not Asian lung cancer patients. Br J Cancer 99: 245-252, 2008.

17. Jala VR, Radde BN, Haribabu B and Klinge CM: Enhanced expression of G-protein coupled estrogen receptor (GPER/GPR30) in lung cancer. BMC Cancer 12: 624, 2012.

18. Sunaga N, Kaira K, Imai H, et al: Oncogenic KRAS-induced epiregulin overexpression contributes to aggressive phenotype and is a promising therapeutic target in non-small-cell lung cancer. Oncogene 32: 4034-4042, 2013.

19. Zhou BB, Peyton M, He B, et al: Targeting ADAM-mediated ligand cleavage to inhibit HER3 and EGFR pathways in non-small cell lung cancer. Cancer Cell 10: 39-50, 2006.

20. Tomshine JC, Severson SR, Wigle DA, Sun Z, Beleford DA, Shridhar V and Horazdovsky BF: Cell proliferation and epidermal growth factor signaling in non-small cell lung adenocarcinoma cell lines are dependent on Rin1. J Biol Chem 284: 26331-26339, 2009.

21. Zito CR, Jilaveanu LB, Anagnostou V, et al: Multi-level targeting of the phosphatidylinositol-3-kinase pathway in non-small cell lung cancer cells. PLoS One 7: e31331, 2012.

22. Khode R, Larsen DA, Culbreath BC, et al: Comparative study of epidermal growth factor receptor mutation analysis on cytology smears and surgical pathology specimens from primary and metastatic lung carcinomas. Cancer Cytopathol 121: 361-369, 2013.

23. Uchiyama M, Usami N, Kondo M, et al: Loss of heterozygosity of chromosome 12p does not correlate with KRAS mutation in non-small cell lung cancer. Int J Cancer 107: 962-969, 2003.

24. Futreal PA, Wooster R and Stratton MR: Somatic mutations in human cancer: Insights from resequencing the protein kinase gene family. Cold Spring Harb Symp Quant Biol 70: 43-49, 2005.

25. Medina PP, Romero OA, Kohno $\mathrm{T}$, et al: Frequent BRG1/SMARCA4-inactivating mutations in human lung cancer cell lines. Hum Mutat 29: 617-622, 2008.

26. Sordella R, Bell DW, Haber DA and Settleman J: Gefitinib-sensitizing EGFR mutations in lung cancer activate anti-apoptotic pathways. Science 305: 1163-1167, 2004.

27. Kobayashi S, Boggon TJ, Dayaram T, et al: EGFR mutation and resistance of non-small-cell lung cancer to gefitinib. N Engl J Med 352: 786-792, 2005.

28. Shibata T, Hanada S, Kokubu A, et al: Gene expression profiling of epidermal growth factor receptor/KRAS pathway activation in lung adenocarcinoma. Cancer Sci 98: 985-991, 2007.

29. Sharma R, Haque AK, Awasthi S, Singh SV, Piper JT and Awasthi YC: Differential carcinogenicity of benzo[a]pyrene in male and female CD-1 mouse lung. J Toxicol Environ Health 52: 45-62, 1997.

30. Paik PK, Arcila ME, Fara M, et al: Clinical characteristics of patients with lung adenocarcinomas harboring BRAF mutations. J Clin Oncol 29: 2046-2051, 2011.

31. Ji H, Wang Z, Perera SA, et al: Mutations in BRAF and KRAS converge on activation of the mitogen-activated protein kinase pathway in lung cancer mouse models. Cancer Res 67: 4933-4939, 2007.

32. Tang X, Shigematsu H, Bekele BN, Roth JA, Minna JD, Hong WK, Gazdar AF and Wistuba II: EGFR tyrosine kinase domain mutations are detected in histologically normal respiratory epithelium in lung cancer patients. Cancer Res 65: 7568-7572, 2005.

33. Hu H, Pan Y, Li Y, Wang L, Wang R, Zhang Y,Li H, Ye T, Zhang Y, Luo X, et al: Oncogenic mutations are associated with histological subtypes but do not have an independent prognostic value in lung adenocarcinoma. Onco Targets Ther 7: 1423-1437, 2014.

34. Bonanno L, Schiavon M, Nardo G, Bertorelle R, Bonaldi L, Galligioni A, Indraccolo S, Pasello G, Rea F and Favaretto A: Prognostic and predictive implications of EGFR mutations, EGFR copy number and KRAS mutations in advanced stage lung adenocarcinoma. Anticancer Res 30: 5121-5128, 2010.

35. Marchetti A, Felicioni L, Malatesta S, et al: Clinical features and outcome of patients with non-small-cell lung cancer harboring BRAF mutations. J Clin Oncol 29: 3574-3579, 2011.

36. Froudarakis ME: Pleural effusion in lung cancer: more questions than answers. Respiration 83: 367-376, 2012. 